\title{
COOPERATIVE INTERACTION OF POLYSACCHARIDE MOLECULES IN WATER : A ROLE OF CONNECTIVITY PROPERTIES OF H-BONDS WITHIN THE SOLVENT?
}

\author{
P.L. San Biagio, F. Madonia, F. Sciortino, M.B. Palma-Vittore1li and \\ M.U. Palma \\ Istituto di Fisica dell'Università, Istituto Applicazioni Interdisciplinani \\ delza Fisica, Cons. Naz. Ric. via Archirafi 36, T-90123 Palermo, Italy
}

Résumé - Nous avons étudié la transition vers un ordre surmoléculaire d'un simple polysaccharide structurel en solution aqueuse et les propriétés structurelles de la phase ordonnée. Nous avons observé des instabilités des propriétés d'agrégation et de transport à travers la transition. Nous discutons le possible rôle de la stéréodynamique du système eau-biomolécule à la lumière du concept de parcours de connexion des liaisons hydrogène.

\begin{abstract}
We study the transition to supramolecular ordering of a simple biostructural polysaccharide in water, and the structural properties of the ordered phase. Instabilities in aggregation and transport properties are observed across the transition. The possible role of the stereodynamics of the biomolecule-water system. as a whole is discussed in terms of the notion of connectivity pathways of hydrogen bonds.
\end{abstract}

\section{I - INTRODUCTION}

Modelling the interplay between the rearrangement of solvent molecules in the presence of solutes and the functional conformation of a biomolecule in water is a very important step towards a deeper understanding of biomolecular stability. To this purpose, an experimental study spanning across a conformational transition of solute biomolecules in a simple two-components aqueous system may prove fruitful. Particularly so if pattern recognition between solute molecules at relatively low soncentration is the initial step of the transition.

As it was suggested long ago /1/ this modelling must include the dynamic domain, in order to account for the second half of the phase space available to the interacting solvent and solute molecules. There has been a very early indication of the interest of taking jointly into account biomolecular dynamics and conformation /2-4/, as a source for the understanding or the prediction of novel phenomena $/ 3 / 4 /$. This has been followed by an increasing amount of interest concerning dynamic aspects of biomolecular physics, their computer simulation, their conjectured or established relevance to functional efficiency, and more in general the role of the time variable, that is dynamics, fluxes and fluctuations $/ 1 / 3-10 /$.

Our modelling could not ignore recent progresses in the understanding of the structure of liquid water in terms of the topologic notion of percolation, as proposed theoretically /11/ and visualized by Molecular Dynamics simulation /12/. The existence of flickering connectivity pathways of $\mathrm{H}$-bonds in water surrounding solutes has been visualized by Monte Carlo /13/ and Molecular Dynamics/12/ simulation. On plain statistical thermodynamic grounds, we have proposed a model wich visualizes at a microscopic level how these topologic structures, as pinned at or modified by different sites at biomolecular surfaces, or as rearranged upon a conformational transition of a solute biomolecule could have a significant role in biomolecular configurational stability and ordering/14/15/.

In this connection, a revisitation of thermoreversible sol-gel transitions in aqueous biomolecular systems appears promising. Difficulties encountered in formulating a u- 
niversal description of these transitions may possibly reflect their sensitivity to molecular details /16/. Similar difficulties and interest concern the dynamic aspects of transitions $/ 17 / 18 /$.

In the present paper we report preliminary results of non-equilibrium studies of correlation spectroscopy across the thermoreversible sol-gel transition of Agarosewater systems. Agarose (M.W. 100,000 - 120,000) is an unbranched and essentially uncharged biostructural polysaccharide which in aqueous solutions forms thermoreversible gels, down to concentrations of $0.3 \% \mathrm{w} / \mathrm{v}$ or less $/ 19 / 20 /$. Gelation is caused by molecular ordering in double helices, followed by partial supramolecular ordering $120 /$, both occurring cooperatively as evidenced by a large thermal hysteresis $/ 21 /$. A Monte Carlo simulation of the interaction at $300^{\circ} \mathrm{K}$ of the Agarose double helix and solvent water has evidenced the occurrence of $\mathrm{H}$-bond connectivity pathways around the double helix /22/. The thermal hysteretical cycle is altered by substitution of $\mathrm{H}_{2} 0$ with $\mathrm{D}_{2} 0$, and the effect appears to be entropic /14/15/21/. Gelation kinetics at different temperatures and up to thousands of hours of observation have also been studied $/ 23 /$. Solvent deuteration causes non-trivial effects. The nature of the transition $/ 24 /$, the time required for it to occur, and the structural characteristics of ge1s so obtained, depend strongly upon the selected temperature and polymer concentration /23/24/. A narrow temperature interval separates two markedly different gelation behaviours /15/23/. In the same interval, or close to it, ample transient fluctuations of the scattered light have been reported to occur in the course of cooling, but they have not been followed up /25/. Light scattering experiments at temperatures below the sol-gel transition have revealed the occurrence of long term, long range fluctuations $/ 24 / 26 /$.

In view of the interest of dynamic studies and also because a sol-gel transition temperature is hardly defined in these systems $/ 23 /$, the present experiments were done in non equilibrium conditions. We have used solvent perturbations for studying the role of solvent in pattern recognition and in molecular organization and dynamics. To this purpose, we have studied across the transition the fluctuations of scattered light intensity in different solvents and compared them to similar fluctuations that we have observed in transport properties as measured non-perturbatively, using minute quantities of polystyrene latex spheres as probes $/ 27 /$. Further information on the role of the solvent was obtained from data on turbidity of gels formed in different conditions, analyzed so as to allow sorting out the effects on the mean square fluctuations of the polymer molar partial volume, and the spatial correlation function of the static fluctuations. We shall discuss our results in terms of the mentioned microscopic model which makes use of the notion of connectivity pathways of H-bonds.

\section{II - EXPERIMENTAL}

Photon scattering and correlation measurements were done with a Malvern RR 102 spectrometer, a Malvern Log-Lin 7027 correlator, and a Coherent 52 Argon Laser operated at $514.5 \mathrm{~nm}$. Data collected with a logarithmic channel spacing were analyzed with the Malvern software which uses the exponential sampling method $/ 28 /$. The same software was used for analysis of data collected in the linear 60-channel mode, but in this case we also used the procedure described in Ref.27, modified so as to allow fittings with two exponential plus a constant whenever necessary. The two different analyses gave results which coincided within a very narrow margin. This added to reliability. In most experimental conditions, diffusion coefficients to be measured ranged within a not so large interval, and advance information was available on the number and dispersivity of the mobile species involved. This, and the presence of a flat background component in the normalized correlogram, made the use of linearly spaced channels and our method of fitting more convenient in the present case. The method of cumulants $/ 29 / 30 /$ was also used occasionally, that is when high polydispersity was expected or possible. Data were taken at a constant $90^{\circ}$ scattering angle, an extension of experiments to various angles being now under way. Photon correlation spectrometry was also used for studying viscosity at a microscopic scale and non-perturbatively. To this purpose we measured the translational diffusion coefficient of monodisperse 
polystirene microspheres of 0.04 micrometer nominal radius, added in minute quantities during preparation of specimens /27/.

Turbidity was measured with a Jasco 505 Uvidec spectrometer. In every experiment, temperature was controlled and scanned with a Hake programmable thermostat and actual1y measured with a Fluke thermometer using a platinum sensor.

Agarose was Seakem HGT (P), lot no.62933, with sulfate contents less than $0.15 \%$. The powder was dissolved in the solvent by keeping the sealed samples in boiling water for about $20 \mathrm{~min}$. Samples were then filtered through 0.2 micron filters, at $70^{\circ}-90^{\circ} \mathrm{C}$. If not differently stated, temperature was then decreased from $70^{\circ} \mathrm{C}$ at the rate of $1.8^{\circ} \mathrm{C} / \mathrm{hr}$. Water was Millipore Super $\mathrm{Q}$ and $\mathrm{D}_{2} \mathrm{O}$ was from Norsk Hydro (Oslo, Norway). Ethanol was standard reagent grade and it was added to solvent water during preparation of samples. A molar fraction of 0.03 Ethanol in water was used as a convenient solvent perturbation/31/. Extension to different alcohols and concentrations is now under way.

\section{III - RESULTS}

Experiments with sols at $70^{\circ} \mathrm{C}$.

An Agarose concentration of $0.5 \% \mathrm{w} / \mathrm{v}$ was chosen for the present study, on the basis of preliminary photon correlation spectrometry (PCS) experiments. Sols at different Agarose concentrations in $\mathrm{H}_{2} \mathrm{O}$ and at $70{ }^{\circ} \mathrm{C}$ were observed. Two exponentials plus a constant (see foregoing Section) gave very satisfactory fittings of correlograms, showing the presence of: i) a paucidisperse species, with hydrodynamic radius of the order of 1,500-2,000 Angstrom, and ii) a similarly paucidisperse species with hydrodynamic radius of the order of 150 Angstrom. This latter value compares favourably with the expected average radius of a random coiled Agarose polymer and does not depend upon polymer concentration, in the range of our measurements. The presence of species having radii similar to type i) has already been reported and discussed in the literature/24/, but to the present purpose we are only concerned with type ii). Non-perturbative measurements of viscosity were also done by PCS as described in the previous section. Results relative to three solvents and different Agarose concentrations are shown in Fig.1, where $\eta$ is the measured viscosity, $\eta$ is the viscosity of the solvent (as measured by the same method), $\left(\eta_{q}-\eta_{0}\right) / \eta_{0}$ is the so called specific viscosity, d is the center-to-center average distance between Agarose molecules, and

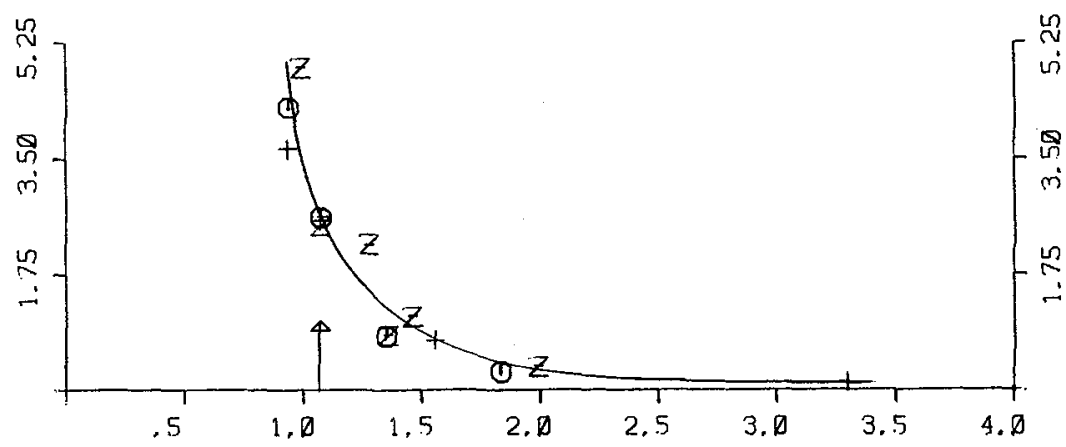

Fig. 1 Specific viscosity vs. $\mathrm{d} / 2 \mathrm{R}$ in Agarose sols at $70{ }^{\circ} \mathrm{C}$ in $\mathrm{H}_{2} \mathrm{O}(0), \mathrm{D}_{2} \mathrm{O}(+)$ and 0.03 Mol. fract. Et-OH solution ( $z$ ). Arrow corresponds to $0.5 \%$ w/v Agarose concentration. 
$\mathrm{R}$ their measured hydrodynamic radius. Note the marked rise in the viscosity at value one in the abscissa, corresponding to the overlap threshold concentration /16/, at which the polymer random coils are just about in contact. The concentration of $0.5 \%$ $\mathrm{w} / \mathrm{v}$ was chosen for the present experiments for its being close to this threshold value. To be noted that points relative to different solvents fall on one and the same curve when plotted vs. the scaled variable $d / 2 R$. This adds reliability to results of our PCS experiments and data analyses.

\section{Instabilities and fluctuations across the sol-gel transition.}

Viscosity and light scattered at $90^{\circ}$ were measured across the sol-gel transition, for a decreasing-temperature scanning rate of $1.8{ }^{\circ} \mathrm{C} / \mathrm{hr}$, starting from $70^{\circ} \mathrm{C}$. Results of a typical run are shown in Fig. 2, where specific viscosity and the normalized number of scattered photons averaged over a $100 \mathrm{sec}$. time basis are plotted against tempera-

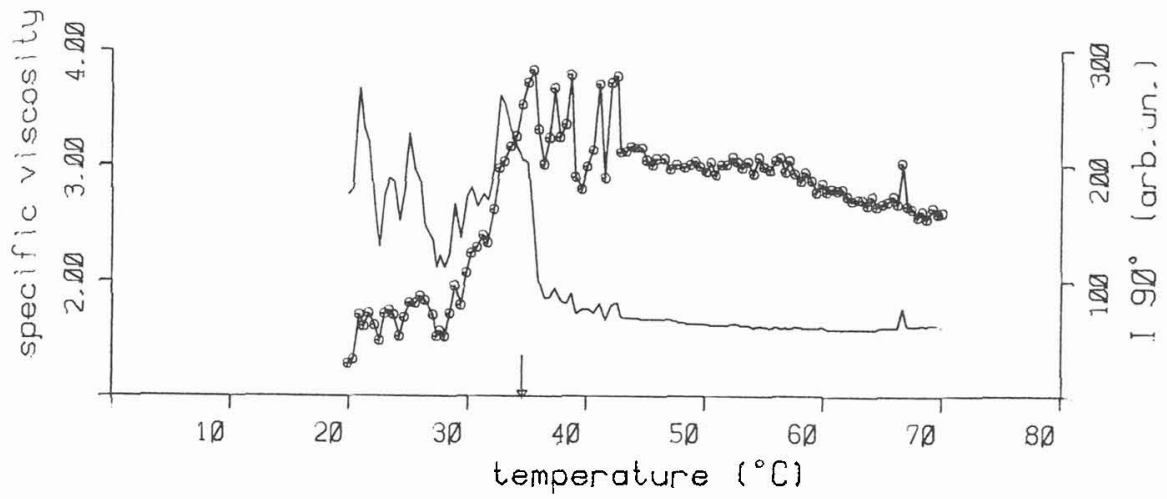

Fig. 2 - Specific viscosity (-o-o-) as measured by 0.04 radius micrometer spheres, and $100 \mathrm{sec}$-average, normalized counts of photons scattered at $90^{\circ}$. Typical run with a sample of $0.5 \% \mathrm{w} / \mathrm{v}$ Agarose in $\mathrm{H}_{2} \mathrm{O}$, and with temperature decreasing at $1.8^{\circ} \mathrm{C} / \mathrm{hr}$. The arrow indicates the temperature of occurrence of the sol-gel transition, as monitored turbidometrically at the same scanning rate.

ture. An aliquote of the same specimen, identically treated, was used for recording the occurrence of the sol-gel transition, as revealed by the steep increase of optical turbidity /21/. At our scanning rate, the width (between $25 \%$ and $75 \%$ of the increase in turbidity) of the transition so measured is about $6{ }^{\circ} \mathrm{C}$ and its $50 \%$ point is indicated on the temperature scale in the Figure. The observed viscosity fluctuations could in principle be due to an artefact caused by the appearance and disappearance of large species, capable of "entraining" the photon correlation function. As it will be discussed elsewhere, all our efforts for finding an explanation of this kind were unsuccessful. This was essentially due to the fact that in our conditions the contribution of polystyrene spheres dominates the correlation function. Results must therefore be ascribed to viscosity fluctuations. Sequences of events in repeated runs at decreasing temperatures in $\mathrm{H}_{2} \mathrm{O}$ systems have in common the following features: i) microscopic viscosity is observed to fluctuate well before the start of the turbidometric gelation curve. Measurements at constant temperature in this range were not attempted, but it is known from other experiments that here an exceedingly slow gelation process occurs, originating a new type of gel structure $/ 23 /$; ii) average value and fluctuation amplitude of specific viscosity start both decreasing when the turbidometrically monitored transition is about at its midpoint; iii) intensity and fluctuations of light scattered at $90^{\circ}$ start instead increasing at about this temperature; iv) the final value of specific viscosity measured by our probes is smaller 
than that relative to sols at $70^{\circ} \mathrm{C}$, indicating that empty spaces in the gel are sizeably larger than our probes $/ 26 / 27 /$.

In $\mathrm{D}_{2} \mathrm{O}$, viscosity fluctuations, turbidometric gelation curve, and scattered light fluctuations all appear at temperatures about $2{ }^{\circ} \mathrm{C}$ higher than in $\mathrm{H}_{2} 0$. However, the temperature at which large viscosity fluctuations decrease is about ${ }^{2}{ }^{\circ} \mathrm{C}$ higher, so that their interval of occurrence is narrowed.

In $\mathrm{H}_{2} \mathrm{O}$ with 0.03 molar fraction Ethanol, the turbidometric gelation curve occurs at a temperature some 2 or $3{ }^{\circ} \mathrm{C}$ lower than in $\mathrm{H}_{2} \mathrm{O}$. The start of viscosity fluctuations is also similarly shifted but they start decreasing at the same temperature as for $H_{2} 0$, with a resulting narrowing of their interval of occurrence. The temperature at which large fluctuations of scattered light intensity appear is not much affected.

Qualitative features of the observed effects of solvent perturbations are such as to make impossible a unified description in terms of the variable $\mathrm{T} / \mathrm{\eta}$ relevant to mass fluxes, or in terms of a shift in temperature, to compensate for the slightly altered strength of hydrogen bond. A combined use of both changes of temperature scale does not appear more viable. As it has already been discussed /15/ and as we shall recall in our conclusions, this is particularly meaningful.

\section{Turbidity spectra of formed gels}

A preliminary study of the role of temperature, Agarose concentration, and solvent in the kinetics of isothermal gelation, containing also partial information on the structural characteristics of the already formed gel, has been published elsewhere /23/. We have prepared a series of gels at $0.5 \% \mathrm{w} / \mathrm{v}$ Agarose, by isothermal gelation at several temperatures and measured their turbidity spectra in the range $350-800$ nm, up to when no appreciable further variation with time was observed. This required waiting times ranging from several hours to several. weeks. For a better comprehension of information contained in the present results, we recall that if $\Phi$ is the partial molar volume of Agarose, turbidity can be expressed in our case as the product of $\left\langle\Delta \Phi^{2}\right\rangle$, which somehow measures the degree of supramolecular aggregation of polymer strands, by a function of the wavenumber of the light propagating in the gel /24/32/. This function is determined by the spatial correlation function of the static fluctuations /32/. As we shall discuss in detail elsewhere, it can be shown that its average slope when represented on a $\log -10 g$ scale is determined by the correlation length.

In Fig.3, a we show spectra of gels obtained in $\mathrm{H}_{2} \mathrm{O}$ at several temperatures. From Fig.3,b again it appears that spectral differences cannot be accounted for by a mere temperature shift. In Fig.3,b turbidity at a given wavenumber is plotted against the temperature at which gels were formed. In account of the constancy of wavenumber, we conclude that turbidity maxima evidence corresponding maxima in mean square fluctuations $\left\langle\Delta \Phi^{2}\right\rangle$. The maximum shifts towards lower temperatures and becomes less pronounced by going from $\mathrm{D}_{2} \mathrm{O}$ to $\mathrm{H}_{2} \mathrm{O}$ to 0.03 molar fraction Ethanol in water. Further, it appears that spectral differences cannot be accounted for by a mere temperature shift. The $\log -\log$ representation in $\mathrm{Fig} \cdot 3, \mathrm{c}$ shows that at 20 and $38^{\circ} \mathrm{C}$ the mean square static fluctuations of the polymer partial molar volume in the formed gel are measurably affected, in the order $\mathrm{D}_{2} \mathrm{O}>\mathrm{H}_{2} \mathrm{O}>\mathrm{EtOH}$, while their correlation functions are less so; and that at $44^{\circ} \mathrm{C}$, both these mean square fluctuations and their correlation functions are markedly affected.

\section{IV - COMMENTS AND CONCLUSIONS}

We have reported for a simple aqueous system of a biostructural polysaccharide, the onset and the evolution of instabilities and fluctuations, across the thermoreversible phase transition that the system undergoes upon cooling. Reference to better known critical phenomena is not straightforward, because the transition exhibits a large thermal hysteresis, the origin of cooperativity is scarcely understood, and the definition of a critical temperature and of an order parameter across the sol-gel transition is not trivial /16/. The reported instabilities and fluctuations, however, 

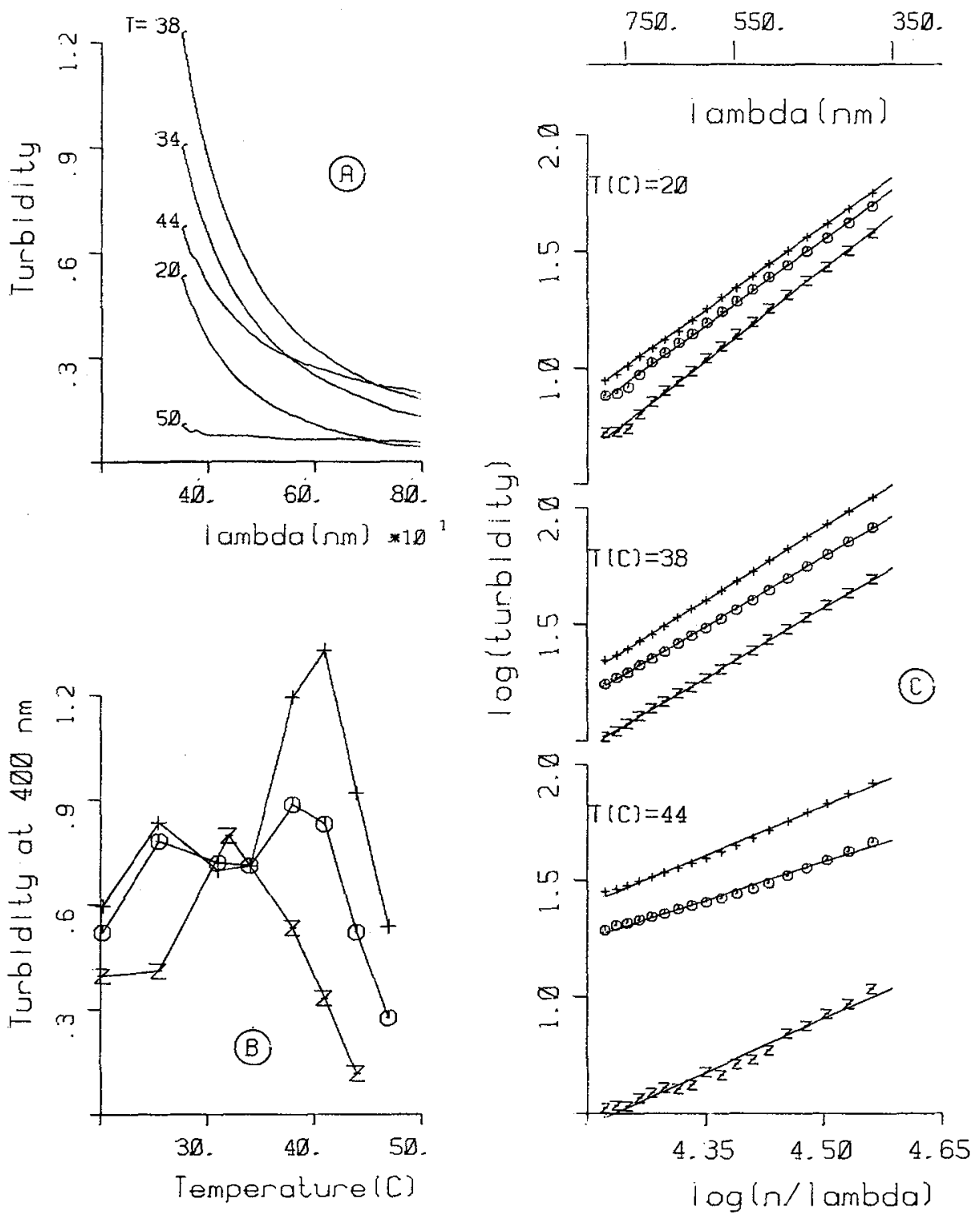

Fig. 3 - Turbidity of $0.5 \% \mathrm{w} / \mathrm{v}$ Agarose gels isothermally formed at selected temperatures.

A) Spectra of $\mathrm{H}_{2} \mathrm{O}$ gels

B) Turbidity at fixed wavelength $(400 \mathrm{~nm})$ of gels vs. temperature at which they were formed: $\mathrm{H}_{2} \mathrm{O}(\mathrm{O}), \mathrm{D}_{2} \mathrm{O}(+), 0.03 \mathrm{Mo}$. fract. Et-OH solution (z)

C) Turbidity of gels formed at three selected temperatures and in three different solvents, vs. wavenumber in the solvent. Symbols as in B). Here $n$ is the index of refraction of the solvent. 
evidence and concern a role of stereodynamics, mass fluxes and hydrodynamics of the entire biomolecule-water system. The behaviour reported here may more generally be typical of transitions to the gel state $/ 27 /$.

In our specific system, transition to the gel state implies a coil-to-helix transformation (with no intrahelical hydrogen bonds) followed by aggregation of helices in bundles /20\%. Results that we have presented suggest that the onset of hydrodynamic/viscometric instabilities be mainly related to the former, and that similar fluctuations in scattered light be instead mainly related to the latter. If so, the present approach possibly offers. for the first time a decoupled study of the two processes.

The two solvent perturbations used here are expected to act in complementary ways, in our model based on the possible role of H-bond connectivity pathways. The mass difference between $H$ and $D$ reduces significantly the librational and vibrational amplitudes in $\mathrm{D}_{2} \mathrm{O}$. It has been suggested that the reduced orientational disorder will facilitate propagation of order along connectivity pathways of hydrogen bonds $/ 14 / 15 /$. This agrees with results of molecular dynamics simulation /33/. On the other hand, addition of monohydric alcohols to the aqueous solvent, in addition to trivial effects due to the altered dielectric constant, is known to alter e.g. the difference between the standard free energies of the two possible conformations of Hemoglobin by an overall, non bulk-electrostatic effect of the solvent as a whole 131\%. This effect can be synthetically thought to be due to a decreased hydrophobic interaction /31/. It might be accounted for in terms of an altered free energy contribution of the H-bonds connectivity pathways, as perturbed by alcohol molecules and their hydrophilic/hydrophobic hydration and interaction /15/. In short, molecular organization and aggregation can be expected to be more efficient in $\mathrm{D}_{2} \mathrm{O}$ and less efficient in $\mathrm{H}_{2} \mathrm{O}$-Ethanol solutions, on the basis of this model.

Solvent perturbation results are summarized as it follows. In $\mathrm{D}_{2} \mathrm{O}: \mathrm{i}$ ) viscometric instabilities occur in a narrower temperature interval, as if helices aggregate more efficiently as soon as they form; ii) amplitude of scattered light fluctuation is larger, as if potential aggregates could be larger in $D_{2} 0$, and $\left.i i i\right)\left\langle\Delta \Phi^{2}\right\rangle$ values are larger, again speaking for a more efficient aggregation. (All this possibly implies some recognition process). At variance, in Ethanol solutions, although fluctuations of scattered light are not delayed in this case with respect to viscosity fluctuations, aggregation as measured by $\left\langle\Delta \Phi^{2}\right\rangle$ appears to be less efficient.

Predictions from the model appear thus confirmed by present data. The model itself, however, needs to undergo much more critical tests and scrutiny. If confirmed, it would in essence amount to saying that an increased stability or a disturbance of the H-bonds connectivity pathways in water may interfere with the coming together of solute biomolecules. Hydrophobic surfaces, inasmuch that they alter the connectivity pattern, would appear capable of interfering with such processes just as well as hydrogen bonding sites.

We express our sincere thanks to Prof. L. Cordone, Prof. S. Fornili and Dr. M. Migliore for several discussions and access to unpublished results; to Mr. F. Ficarra, Mr. F. Nicotra, Mr. M. Lapis and Mr. A. La Gattuta for valuable technical help, and to Ms. M. Genova-Baiamonte and Ms. M. Giannola for producing the processed typescript. The present work was supported by Consiglio Nazionale delle Ricerche (Institute for Interdisciplinary Applications of Physics), by CRRNSM (Palermo) and by M.P.I. local fundings.

\section{REFERENCES}

(1) Aiello, G., Giammarinaro, M.S., Palma-Vittorelli, M.B., Palma, M.U. in "Cooperative Phenomena", edited by Haken, H., Wagner,M., Berlin, 1973, p. 395. Palma M.U., in "From Theoretical Physics to Biology" edited by Marois M., Basel, 1973, p. 21 . 
(2) Linderstrøm-Lang, K.U., Schellman, J.A. in "The Enzymes" edited by Boyer, D., Lardy, H., Myrbach, K., New York, 1959, vol. 1, p. 12.

(3) Fröhlich, H., in "Theoretical Physics and Biology" edited by Marois, M., Amsterdam, 1969, p. 13, and Adv. Electronics and Electr. Phys. 53 (1982) 85.

(4) Fröhlich, H. and Kremer, F., "Coherent Excitations in Biological Systems" (Berlin Heldelberg) 1983.

(5) Careri,G., in "Quantum Statistical Mechanics in the Natural Sciences", edited by Kursunoglu, B., Mintz, S.L., Widmayer, S.M., New York, 1974, p. 37.

Careri, G., Fasella, P., Gratton, E., Ann. Rev. Biophysics Bioengineering, 8 (1979) 69.

(6) Austin, R.M., Beeson, K.W., Eisenstein, L., Frauenfelder, H., Gunsalus, I.C., Biochemistry, 14 (1975) 3555 .

Frauenfelder, H., in "Mobility and Function in Proteins and Nucleic Acids", CIBA Foundation Symposium 93, London, 1982, p. 64.

(7) Davydoff, A.S., "Biology and Quantum Mechanics" (Pergamon Press, Oxford) 1982. Scott, A.C., Phys. Rev. A. 26 (1982) 578.

(8) Englander, S.W., Kallenbach, N.R., Heeger, A.J., Krumhans1, J.A., Litwin, S., Proc. Natl. Acad. Sci. (USA) 77 (1980) 7222.

(9) Karplus, M., Mc Cammon, K., Nature 277 (1979) 578.

(10) San Biagio, P.L., Vitrano, E., Cupane, A., Madonia, F., Palma, M.U., Biochem. Biophys. Res. Comm. 77 (1977) 1158.

(11) Stanley, H.E., Teixeira, J., J. Chem. Phys. 73 (1980) 3404.

(12) Stillinger, F.H., Science 209 (1980) 451.

(13) Clementi, E., Barsotti, R., Fromm, J., Watts, R.0., Theoret. Chem. Acta (Ber1.) 43 (1976) 101 .

Fromm, J., Clementi, E., Watts, R.0., J. Chem. Phys. 62 (1975) 1388; ibid., 61 (1974) 2550.

(14) Palma, M.U., in. "Coherent Excitations in Biological Systems" edited by Fröhlich, H., Kremer, F., Berlin Heidelberg, 1983, p. 84.

Palma, M.U., in "Structure and Dynamics : Nucleic Acids and Proteins" edited by Clementi, E., and Sarma, R.M., Guilderland, N.Y., 1983, P. 125.

(15) Fornili, S.L., Leone, M., Madonia, F., Migliore, M., Palma-Vittorel1i, M.B., Palma, M.U., San Biagio, P.L., J. Biomol. Str. Dyn. 1 (1983) 473

(16) De Gennes, P.G., "Scaling Concepts in Polymer Physics" (Cornell University Press, Ithaca, N.Y.) 1979

(17) Ma, S.K., "Modern Theory of Critical Phenomena" (W. A. Benjamin, Inc., Reading, Mass.) 1976

(18) Stanley, H.E., "Introduction to Phase Transitions and Critical Phenomena" (Clarendon Press. Oxford) 1971

(19) Rees, D.A., Adv. Carbohydr. Res. 24 (1969) 267

(20) Arnott, S., Fulmer, A., Scott, W. E•, Dea, I.C.M., Moorhouse, R., Rees, D.A., J. Mol. Biol. 90 (1974) 269

(21) Vento, G., Palma, M.U., Indovina, P., J. Chem. Phys. 70 (1979) 2848; ibid. p. 2841.

(22) Corongiu, G., Fornili, S.I., Clementi, E., Int'1 J. Q. Chem., Q. Biol. Symp. 10 (1983) 277 .

(23) Leone, M., Fornili, S.L., Palma-Vittorel1i, M.B., in "Water and Ions in Biological Systems : 2nd International Conference" edited by Vasilescu, V., Pullman, B., Packer, L., Leahu, L., London, 1984, p. 103.

(24) Wun, K.I., Feke, G.T., Prins, W., Faraday Disc. Chem. Soc. 57 (1974) 146.

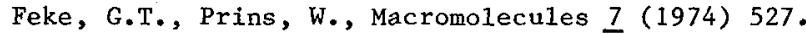

(25) Letherby, M.R., Young, D.A., J. Chem. Soc. Faraday Trans. 1, 77 (1981) 1953.

(26) Sellen, D.B., Polymer 19 (1978) 1110. Mackie, W., Sellen, D.B., Sutcliffe, J., Polymer 19 (1978) 9. Key, P.Y., Sellen, D.B., J. Polymer Sci. : Polymer Phys. Edition 20 (1982) 659.

(27) Madonia, F., San Biagio; P.L., Palma, M.U., Schiliro', G., Musumeci, S., Russo, G. Nature 302 (1983) 412 .

(28) Ostrowsky, N., Sornette, D., Parker, P., Pike, E.R., Optica Acta 28 (1981) 1059.

(29) Koppel, D.E., J. Chem. Phys. 57 (1972) 4814.

Pusey, P.N., Koppel, D.E., Schaefer, D.W., Camerini-Otero, R.D., Koenig, S.H., I.B.M. Res. Rept. RC 3924 (Yorktown Heights, N.Y., 1972). 
(30) Brown, J.C., Pusey,P.N., Dietz,R., J.Chem.Phys. 62 (1975) 1136.

(31) Cordone, L., Cupane, A., San Biagio, P.L., Vitrano, E., Biopolymers 18 (1979) 1975.

Cordone, L. Cupane, A., San Biagio, P.L., Vitrano, E., Biopolymers 20 (1981) 39 and ibid. 20 (1981) 53.

Cordone, L., Cupane, A., Biopolymers 20 (1981) 2137.

Cupane, A., Giacomazza, D., Cordone, L., Biopolymers 21 (1982) 1081.

Cordone, L., Cupane, A., D'Alia, F., De Stefano, M.G., Faraday Symp. Chem. Soc. (London) 17 (1982) 161 .

Cordone, L., Cupane, A., Fornili, S.L., Biopolymers 22 (1983) 1677.

Bulone, D., Cupane,A., Cordone, L., Biopolymers 22 (1983) 119.

(32) Pines, E., Prins, W., Polymer Letters 10 (1972) 719.

(33) Hirata, F., Rossky, P.J., J.Chem.Phys. 74 (1981) 6867 